Ferramentas Tecnológicas Disponíveis Gratuitamente para Uso no Ensino de Química: Uma Revisão

\author{
Ferreira, M. P.;* Suzuki, R. M.; Bonafe, E, G.; Matsushita, M.; Berton, S.
}

B. R.

Rev. Virtual Quim., 2019, 11 (3), 1011-1023. Data de publicação na Web: 1 de julho de 2019

http://rvq.sbq.org.br

\title{
Technological Tools Available Free for Use in Chemistry Education: A Review
}

Abstract: The digital tools are promissory to the teaching chemistry at the high school and undergraduate levels because we believe that by implanting these technologies are produced a better teaching-learning. However, it is known that digital tools are often not used in the school and university routine due to the lack of knowledge by teachers, as well by the lack of support offered by the educational institutions. In this context, this work envisions to present information and communication technologies (ICTs), both in a specific and generalized approach, which can be used for teaching Chemistry. This may contribute to improve the formation and thereby provide better teachers. It was carried out a bibliographic survey by using national and international journal sources. Thirty-seven papers are selected for the elaboration of this study. The results showed that by applying the ICTs can help the understanding of contents proposed in classrooms. Furthermore, the ICTs instigate the students, generating better participation and interest, making a more comfortable and dynamic environment for a better teaching-learning. In this facet, it is interesting that teachers use such technologies to promote meaningful, diversified, and evolutionary learning.

Keywords: Educational software; applications; academic training; ICTs.

\section{Resumo}

As ferramentas digitais são promissoras ao ensino de Química tanto em nível médio como superior, acreditando-se que a implantação dessas tecnologias produz avanços no ensino-aprendizagem. Sabe-se, porém, que ainda é pouco utilizada na rotina escolar pela falta de conhecimento por parte do docente e pela falta de suporte oferecido pela instituição de ensino. Neste contexto, este trabalho tem como objetivo apresentar tecnologias de informação e comunicação (TICS), tanto de forma específica como generalizada, que podem ser utilizadas no ensino da Química, com intuito de contribuir na formação dos docentes e futuros docentes. Para tal, foi feito um levantamento bibliográfico nas principais plataformas de periódicos nacionais e internacionais. Desse levantamento, foram selecionados trinte e sete artigos para a elaboração desse estudo. Os resultados obtidos na presente pesquisa, mostraram que a utilização das TICs favorece a compreensão dos conteúdos propostos em sala de aula, além de instigar a participação e o interesse dos alunos tornando o ambiente descontraído e dinâmico. Dessa forma, é conveniente que o professor usufrua dessa tecnologia que está sempre à mão do aluno, a fim de promover uma aprendizagem significativa, diversificada e evolutiva.

Palavras-chave: Softwares educativos; aplicativos; formação acadêmica; TICs.

\footnotetext{
* Universidade Estadual de Londrina, Departamento de Química, CEP 86057-970, Londrina-PR, Brasil.

Milenapf41@hotmail.com

DOI: $\underline{10.21577 / 1984-6835.20190068}$
} 


\title{
Ferramentas Tecnológicas Disponíveis Gratuitamente para Uso no Ensino de Química: Uma Revisão
}

\section{Milena do Prado Ferreira, ${ }^{\mathrm{a},{ }^{*}}$ Rúbia Michele Suzuki, ${ }^{\mathrm{b}}$ Elton Guntendorfer Bonafe, ${ }^{\mathrm{b}}$ Makoto Matsushita, ${ }^{\mathrm{c}}$ Sharise Beatriz Roberto Berton ${ }^{\mathrm{c}}$}

\author{
a Universidade Estadual de Londrina, Departamento de Química, CEP 86057-970, Londrina-PR, \\ Brasil. \\ b Universidade Tecnológica Federal do Paraná, Departamento de Química, Campus Apucarana, \\ CEP 86812-460, Apucarana-PR, Brasil. \\ c Universidade Estadual de Maringá, Departamento de Química, Maringá-PR, Brasil. \\ * milenapf41@hotmail.com
}

Recebido em 1 de fevereiro de 2019. Aceito para publicação em 29 de maio de 2019

\section{Introdução}

\section{Metodologia}

\section{Levantamento Bibliográfico}

3.1. Software de simulação

3.2. Ferramentas de modelização

3.3. Podcast e vodcast

3.4. Aplicativos educacionais para smartphone

3.5. Blogs e redes sociais

\section{Discussão}

5. Conclusão

\section{Introdução}

A educação possui como finalidade preparar os indivíduos para o exercício de uma profissão, incentivar a prática científica e civilizar, ou seja, preparar as pessoas para a sociedade, formando seres críticos com uma consciência político-social e que exerçam assim a cidadania. O conhecimento e a capacidade de aprendizado ao longo da vida são condições necessárias para o desenvolvimento humano. ${ }^{1,2}$

Cada vez mais, o modelo pedagógico vem sofrendo mudanças, visto a importância e a necessidade da atualização frente as novas demandas que o mercado exige. A rapidez com que a tecnologia se desenvolve também contribui para essa nova perspectiva, pois as mesmas trouxeram novos horizontes e possibilidades. $^{3}$

A tecnologia é uma ferramenta poderosa que facilita a aprendizagem e estimula a 
cognição de alunos e professores, pelo grande poder de alcance e pelo fato de que a tecnologia invade nossa vida e as residências mais distantes do mundo. Podemos definir tecnologia como sendo o conjunto de instrumentos, métodos e técnicas que visam a resolução de problemas.

A partir do século XX, destacam-se as TICs (Tecnologias de Informação e Comunicação) através da evolução das telecomunicações, utilização dos computadores e desenvolvimento da internet. Consiste de todos os meios técnicos usados para tratar a informação e auxiliar na comunicação, o que inclui o hardware de computadores, rede, telemóveis, bem como todo software necessário. ${ }^{4-6}$ Elas fazem parte da realidade sociocultural dos estudantes, dessa forma precisam ser consideradas nos processos de mediação pedagógica, pois interferem nas formas de acessar a informação e aprender. ${ }^{7}$

Nesse sentido, tem-se as TICs como intermediadoras no processo de ensino/aprendizagem, possibilitando alterações pedagógicas e a criação de um novo paradigma educacional. Como a tecnologia está fortemente presente, é de extrema importância levar em consideração a sua implementação na educação. Com diversas possibilidades de aplicação, cria-se uma oportunidade ímpar para os professores repensarem sua prática educativa. ${ }^{8,9}$

É possível citar diversas ferramentas tecnológicas que auxiliam os ambientes educacionais, tanto em comunicação síncrona como na assíncrona: recursos audiovisuais, multimídia interativa, correio eletrônico ( $e$ mail), grupos de discussão, world wide web WWW, download, vídeo e áudio sob demanda, chat, webquest, podcast, vodcast, blogs, simuladores, recursos da internet (livros eletrônicos, periódicos eletrônicos, bibliotecas virtuais, curso a distância), YouTube, Facebook, WhatsApp, jogos lúdicos online, aplicativos para smartphone, entre muitos outros. Uma boa parte dos recursos online e de softwares são gratuitos, oferecendo ao professor um leque de possibilidades para trabalhar certo conteúdo. ${ }^{10}$
Ao longo das três últimas décadas, os softwares educacionais vêm sendo amplamente utilizados como apoio no ensino de Química, sendo categorizados como: jogos educacionais, exercício e prática (exercícios ou questões para o aluno resolver), cálculo computacional (resolvem equações matemáticas), aquisição de dados e análise de experimentos (voltados para análises experimentais, traçando gráficos e tabelas estatísticas diferentes), tutorial (apresentação de determinado conteúdo de forma mais dinâmica), produção de gráficos, caracteres especiais, entre outros. ${ }^{11-13}$

Assim, as ferramentas tecnológicas podem atuar como auxiliadoras em temas abstratos no ensino de Química, nos quais os alunos apresentam maior dificuldade em compreender e reter certo conhecimento. ${ }^{13,14}$

É importante que o professor, nesse novo cenário, tenha conhecimento de como manipular essas novas tecnologias, tornando mais efetiva a participação dos alunos e proporcionando diferentes meios de aprendizagem. $\mathrm{O}$ conhecimento prévio dos discentes e o contato direto com a tecnologia contribui para a implantação desses recursos tecnológicos, pois manusear programas e navegar em sites são práticas do cotidiano deles. ${ }^{15,16}$

Diante disso, este trabalho tem como objetivo apresentar os recursos já disponíveis, mostrando que é possível integrar o ensino ao uso dessas ferramentas digitais, visando seus efeitos sobre a aprendizagem dos alunos, bem como motivar o seu uso no ensino de Química.

\section{Metodologia}

Inicialmente, a pesquisa ocorreu por meio do levantamento prévio de artigos que abordam a utilização das tecnologias como ferramenta de apoio no processo de ensino e aprendizagem no ensino de Química. A referida pesquisa, de revisão bibliográfica, foi realizada utilizando bases científicas como ISI - Web of Science, Scielo e Portal da Capes, por meio das seguintes palavras-chave: software 
educacional, tecnologias digitais, ensino de química, recursos tecnológicos, ensinoaprendizagem, tecnologia da informação e as expressões equivalentes a estas palavraschave na língua inglesa, tais como educational software, digital technology, chemistry teaching, technological resources, teaching and learning, information technology. Ao todo, foram identificados cinquenta artigos que os autores julgaram ser relevantes para a temática do estudo. Dentre esses, trinta e sete artigos foram selecionados para a realização do presente trabalho, por serem mais recentes. O critério de escolha deu-se principalmente para os que utilizaram recursos de fácil acesso e que apresentaram diferentes tecnologias de informação e comunicação.

O trabalho de revisão bibliográfica é relevante do ponto de vista científico, por apresentar caráter exploratório, 0 que possibilita maior aprofundamento do assunto abordado, desenvolvido com base em material já elaborado como artigos, livros e teses. ${ }^{17}$

\section{Levantamento Bibliográfico}

A seguir são apresentados diversos recursos tecnológicos que podem ser empregados como auxiliadores na aprendizagem no ensino de Química.

\subsection{Software de simulação}

Os softwares de simulação no ensino de Química ajudam muito no entendimento de fenômenos de nível macroscópico e, principalmente, microscópico, sendo esta segunda opção a que mais dificulta a aquisição de compreensão por parte dos alunos. ${ }^{16,19}$

São programas que trazem modelos de um sistema ou processo, e são classificados em conceituais (retratam conceitos e fatos relacionados ao evento simulado, como por exemplo: a simulação da estruturação de uma molécula) ou operacionais (representam a sequência de operações e procedimentos, como por exemplo: o manuseio de um equipamento usado pra determinado experimento). ${ }^{18}$

Mesmo não sendo projetados para que os alunos os modifiquem, proporcionam um tipo de interação com o programa que permite ao aluno visualizar eventos que acontecem em nível não visível a olho nu, permitindo construir, posteriormente, um modelo mental do fenômeno microscópico, fazendo assim suas próprias inferências e previsões. ${ }^{19}$

Existem softwares de simulações específicos para o currículo de Química, com a possibilidade de aplicação nas áreas de química analítica, bioquímica, cristalografia, farmacologia, físico-química, química geral, química inorgânica, química orgânica, organometálicos e polímeros. Alguns exemplos de softwares de simulação disponíveis para download gratuito são: Avogadro, Molden, Orca e Chimera. ${ }^{18}$

Lima ${ }^{19}$ relata o uso do software Chimera na disciplina de Bioquímica do curso de Licenciatura em Química, a qual trata de assuntos como: estrutura e propriedades da água, aminoácidos, peptídeos, proteínas, ácidos nucleicos, lipídeos e vitaminas. Cada aluno escolheu uma proteína para trabalhar, sendo algumas delas: colágeno, insulina humana, betalactoglobulina (proteína do soro do leite), hemoglobina, pepsina, queratina, entre outras. Os resultados obtidos no trabalho mostram que com o software foi possível uma melhor visualização, manipulação e compreensão de biomoléculas, contribuindo assim para a aprendizagem do conteúdo proposto.

\subsection{Ferramentas de modelização}

Uma outra forma de minimizar a abstratividade dos conteúdos na área de Química é o uso de ferramentas de modelização. São programas em que o usuário 
desenvolve sua própria simulação computacional. Nesse caso, o aluno passa a ser sujeito ativo do seu próprio processo de aprendizagem. Os alunos podem descrever relações entre conceitos, aplicar os modelos construídos e comparar os resultados obtidos com experimentos laboratoriais. Porém, ainda é uma ferramenta pouco explorada pelos professores, assim como os softwares de simulação. O não uso nem sempre é por desconhecerem esses tipos de ferramentas, mas, por não saberem manusear os programas. ${ }^{13,20,21}$

Um estudo feito por Ramos et al., ${ }^{22}$ utilizando esse tipo de programa para modelagem molecular, foi aplicado na turma de Licenciatura de Química, na qual foram selecionados, aleatoriamente, 20 acadêmicos, que possuíam na grade curricular a disciplina de Introdução à Informática, para garantir assim que o aluno tivesse familiaridade com o computador. No trabalho, foram utilizados os programas ChemSketch e HyperChem para proporcionar um melhor entendimento dos mecanismos de reações orgânicas.

Realizou-se a aula expositiva, abordando as origens, os conceitos e aplicações da Química Computacional e da modelagem molecular, assim como, os conceitos da reação de Diels-Alder (reação análoga de um 1,3-butadieno com etino produzindo um 1,4ciclohexadieno - um polímero). Em um outro momento, foi demostrado as reações estudadas através de desenhos em 2D e 3D, através do uso dos programas ChemSketch e HyperChem, respectivamente. Os dados obtidos foram analisados por meio da aplicação de questionários (pré e pósintervenção). Segundo os estudantes, não houve dificuldades em manusear os programas, mesmo muitos não conhecendo os softwares. O esclarecimento do conteúdo abordado e a satisfação em relação ao curso de modelagem foi de $100 \%$ por parte dos envolvidos. Por este motivo, disciplinas que abordam a química computacional, são de extrema importância, pois desenvolvem no aluno a capacidade de simular e visualizar o abstrato.
Raupp, Serrano e Moreira ${ }^{23}$ fizeram uso do software ChemSketch para construção de modelos moleculares no ensino de isomeria geométrica. A primeira etapa consistiu em aplicar um teste para 6 universitários, os quais tinham que representar em três dimensões as formas cis/trans dos seguintes compostos: cis2-buteno e trans-2-buteno; cis-1,2dibromociclopropano e trans-1,2dibromociclopropano; ácido cis-butenodioico (maleico) e ácido trans-butenodioico (fumárico).

Foram disponibilizados os seguintes materiais para desenhar as estruturas: folhas de desenho, régua, lápis, borrachas, canetas esferográficas, conjunto de lápis de cor com 12 cores e um conjunto de canetas hidrográficas com 12 cores. No segundo momento, foi ensinado como manusear a ferramenta de modelização, sendo possível visualizar a estrutura em 3D manipulável pelos estudantes.

Ao final, posteriormente ao uso do software, foi aplicado um segundo teste. Com os mesmos materiais fornecidos anteriormente, os alunos tiveram que representar novamente, através de desenhos, os mesmos pares de isômeros. Observaram, com o estudo realizado, que antes de apresentarem o programa ChemSketch, apenas foram apresentados desenhos na fórmula estrutural e em 2D, a representação tridimensional sequer foi tentada pelos alunos. No entanto, após a apresentação do software, segundo os estudantes, a representação das estruturas ficou mais fácil e foi possível desenhar as estruturas em 3D.

\subsection{Podcast e vodcast}

O podcast surge como uma tecnologia alternativa de apoio ao ensino tanto na modalidade a distância como presencial. Surgiu como o acrônimo das palavras "public on demand" e "broadcast". O termo podcast pode ser descrito de forma resumida como sendo uma emissão pública segundo uma demanda. Assemelha-se a uma subscrição de 
uma revista em áudio e/ou vídeo que podemos receber através da internet. Foi criado em 2004 pelos programadores Dave Winer e Adam Curry, com o propósito de difundir conteúdos diversificados em forma de publicação de ficheiros de multimídia. ${ }^{24}$

Encontram-se podcasts com informações multimídia (slides, fotos e imagens) ajudando seus usuários ampliarem sua percepção sobre o tema abordado. Além disso, um dos seus benefícios é sua compatibilidade em dispositivos portáteis, proporcionando uma comodidade em relação ao seu uso. ${ }^{25}$

Atualmente, são oferecidos outros formatos, como a disponibilização de vídeos nos podcast. Esses são denominados de vodcast (videocast ou vídeo podcast), para especificar conteúdos compostos por vídeo e não apenas por áudio. ${ }^{25}$

Sabe-se que o ser humano absorve e retém certa informação de formas diferentes e que o processo de aprendizagem é composto de características cognitivas, afetivas e psicológicas. Um dos modelos mais conhecidos pelos professores é o de Walter Burke Barbe, que sugere três modalidades de aprendizagem - visual, auditiva e cinestésica. O aprendiz visual possui hábito de observar e identificar cores, desenhos e imagens, possuindo uma memória fotográfica predominante. Tende a olhar a situação de forma panorâmica para depois focalizar os detalhes. O auditivo se identifica muito com sons. Possui habilidades de conhecer, interpretar e diferenciar os estímulos recebidos pela palavra falada, organizando suas ideias, conceitos e abstrações a partir dela. Geralmente lê textos em voz alta e muitas vezes possui talentos musicais. Por fim, o cenestésico não é um bom leitor, prefere ir logo para ação, gosta de se mover, de tocar, de fazer o que é proposto, gesticula enquanto fala e não é bom ouvinte. ${ }^{26}$

Nesse contexto, o podcast torna-se uma ferramenta auxiliadora principalmente para pessoas que possuem uma melhor aprendizagem auditiva, além disso, torna-se um instrumento motivador, já que cria um ambiente de aprendizagem diferente do tradicional.

Hoje, a inclusão escolar é inquestionável, e é dever das instituições de ensino proporcionar condições favoráveis de aprendizagem a portadores de deficiência, a fim de integrá-los na comunidade. Nesse âmbito, a utilização dessa tecnologia é também útil para quem tem alguma necessidade especial, como no caso de alunos com limitações visuais. Dessa maneira, este tipo de mídia não é simplesmente um recurso didático, pois influencia decisivamente no modo com que se constrói o conhecimento. ${ }^{27}$

No trabalho de Leite, Leão e Andrade ${ }^{25}$ é apresentada a criação de um videocast sobre pilhas eletrolíticas com intuito de disponibilizá-lo para uso dos professores de ensino médio e superior. O videocast é composto incialmente por uma breve interação dos apresentadores com os ouvintes. Em seguida, um breve esclarecimento sobre pilhas eletroquímicas e conceitos de reações de óxi-redução foi apresentado através de um exemplo prático. Foi feita a montagem passo a passo de uma pilha eletrolítica $\left(\mathrm{Zn}^{0}+\mathrm{CuSO}_{4} \rightarrow \mathrm{ZnSO}_{4}+\mathrm{Cu}^{0}\right)$, posteriormente foi dado um exemplo de pilha eletrolítica usando duas laranjas, clipes, pedaços de fio de cobre e um relógio digital com a finalidade de comprovar a passagem do fluxo de elétrons pelo sistema. O trabalho elaborado disponibilizou dessa forma, mais um recurso para uso na explicação de pilhas eletroquímicas, através de um exemplo prático, simples e de baixo custo, podendo ser utilizado de forma introdutória ou de revisão em aulas sobre esse assunto.

Em outro momento, Leite ${ }^{28}$ faz uma proposta diferente, quando seleciona 21 professores de Química do curso de Especialização em Ensino de Química, na disciplina de Informática aplicada ao Ensino de Química e propõe que esses elaborem podcasts de Química, os quais são posteriormente avaliados por eles mesmos. Foram sugeridos três podcasts tendo como títulos: Água oxigenada, Efeitos do Álcool e Densidade do Gelo, com duração entre dois a 
quatro minutos cada um deles. Quando questionados sobre: "Quais melhorias na aprendizagem você acredita que o Podcast pode proporcionar?", teve-se como resposta: "O podcast, sendo interativo, contribui para uma boa aprendizagem", "a aprendizagem, utilizando o podcast de maneira ativa é mais eficaz que uma aprendizagem passiva". Os resultados obtidos mostram que o uso do podcast é uma ferramenta atrativa e interativa, podendo contribuir na explanação de conteúdo da Química de forma mais descontraída.

Alguns podcast disponíveis na internet, associados a conteúdos de Química, são:

- Quimica.net

http://www.quimica.net/emiliano/vestibular. html (site repleto de podcast sobre química voltado para as pessoas que pretendem passar no vestibular);

- player FM

https://player.fm/series/chemistry-in-itselement-1244216;

- nature

http://www.nature.com/chemistry/podcast.h tml (especificamente para a comunidade química, contém entrevistas com os vencedores do Prêmio Nobel, discussões sobre pesquisa em nanotecnologia e muito mais);

- ChemiCast

http://www.aulasdequimica.com.br/category /podcast/;

- $\quad$ rsc.org - http://www.rsc.org/periodictable/podcast (disponibiliza podcast sobre a tabela periódica, basta clicar no elemento químico para saber seu significado);

- Portal

Clickideia

https://www.youtube.com/user/PortalClickid eia (disponibiliza não apenas conteúdos voltados para química, mas também para geografia, filosofia entre outras áreas).

\subsection{Aplicativos educacionais para smartphone}

Os dispositivos móveis (Smartphones, Ereaders, Tablets e outros) são a modalidade de TIC mais utilizada, tornando-se uma ferramenta de grande potencial para melhoria e expansão da aprendizagem. ${ }^{29}$

Aplicativo para celular, aplicativo móvel ou simplesmente apelidado como "App" (que vem do inglês application), podem ser gratuitos ou pagos. São programas para celulares, tendo como função entretenimento (jogos, sites de notícias, Netflix), fonte de pesquisa (Google, Yahoo), meio de comunicação (WhatsApp, Facebook, Outlook), aprendizagem (idiomas, culinária, educação escolar), entre outros. ${ }^{30}$

$\mathrm{Na}$ literatura encontra-se o trabalho de Silva, Silva e Silva, ${ }^{31}$ no qual os autores criaram o aplicativo "Studylab", que explora o estudo dos materiais de laboratório, e apresenta 45 ilustrações em ordem aleatória, como a imagem de um almofariz, pipeta volumétrica, pissete, proveta, chapa de aquecimento, entre outros; além das funções de cada utensílio e vídeos de como utilizá-los. Esse aplicativo foi apresentado na forma livre e gratuita, necessitando da internet para a sua utilização. $O$ objetivo do aplicativo era auxiliar os alunos a se familiarizarem com os materiais básicos de laboratório. Foi proposto para quatro professores de ensino médio utilizarem esse aplicativo em sala de aula e observarem se ele contribuiria no processo de ensino e aprendizagem. Ao final, obteve-se a satisfação dos professores na utilização do aplicativo, os quais relataram que o "Studylab" pode ser usado como ferramenta auxiliadora e, em escola que há falta de laboratórios físicos, contribuiria para o aluno conhecer e entender a função dos materiais de laboratório, assim como a demonstração em vídeo da realização de alguns experimentos.

No trabalho de Oliveira, Souto e Carvalho, ${ }^{32}$ foi feito o levantamento de 138 Apps, selecionados na loja virtual para Android, disponível para Smartphones e Tablets, dos quais 9 foram separados pelos critérios: gratuito, nível de satisfação do usuário, idioma português e/ou espanhol e conteúdos relacionados com a Química 
Orgânica, em especial, hidrocarbonetos. Os apps foram: QuimTest, Química Orgânica I, Moléculas, Book, Substâncias Químicas, Fisica\&Química, Hidrocarbonetos, Funções Orgânicas e Nomenclatura. Entre os 9 Apps, os que receberam maior destaque, a partir de diversas características, entre elas a interatividade e potencial para serem integrados a ações docentes no contexto de ensino de Química Orgânica, foram:

- Nomenclatura Orgânica

(https://play.google.com/store/apps/details? id=german.nomenclatura_organica\&hl=pt_B $\mathrm{R})$;

- Química Orgânica I

(https://play.google.com/store/apps/details? $\mathrm{id}=$ com.net.quimicaorganica\&hl=pt_BR);

- Moléculas

(https://play.google.com/store/apps/details? id=|te.ib.unicamp.br.moleculas\&hl=pt_BR);

- Hidrocarbonetos

(https://play.google.com/store/apps/details? id=com. asmolgam.hydrocarbon) e

- Funções orgânicas.

No artigo de revisão bibliográfica, no período de 2011 - 2015 de Nichele e Canto, ${ }^{33}$ os autores filtraram 7 aplicativos. Os aplicativos foram divididos em duas categorias, "estratégias de ensino que utilizam Apps 'Gerais' e estratégias de ensino que utilizam Apps de Química." Na primeira, são os Apps que não foram criados para o ensino de química, mas que podem ser úteis para o desenvolvimento de estratégias de ensino e aprendizagem dessa disciplina. Para os Apps "Gerais", são descritos:

- Notability, disponível apenas para

iPhone e iPad, encontrado no iTunes: https://itunes.apple.com/br/app/notability/i d360593530? $\mathrm{mt}=8$;

- Bamboo Paper, disponível na play store: https://play.google.com/store/apps/details?i $\mathrm{d}=$ com. wacom. bamboopapertab\&hl=pt_BR, e no iTunes: https://itunes.apple.com/br/app/bamboopaper/id443131313? $\mathrm{mt}=8$;

- Panopto, disponível na play store: https://play.google.com/store/apps/details?i $\mathrm{d}=$ com. panopto.androidapp\&hl=pt_BR, e no iTunes:

https://itunes.apple.com/br/app/panopto/id 505718507? $\mathrm{mt}=8$.

Entre o Apps específicos de química, foram destacados:

- Moléculas

(https://play.google.com/store/apps/details? id=Ite.ib.unicamp.br.moleculas\&hl=pt_BR);

- iMolview

(https://play.google.com/store/apps/details? id=com.molsoft.imolview\&hl=pt_BR);

- $\quad$ K12 Periodic Table

(https://play.google.com/store/apps/details? id=air.com.k12. periodictablev2) e

- SocrativeTeacher (https://play.google.com/store/apps/details? $i d=$ com. socrative.teacher).

Os dois primeiros proporcionam a representação e "manipulação" de estruturas tridimensionais de substâncias químicas. Já no terceiro, tem-se uma tabela periódica interativa, enquanto o último exemplo baseiase em perguntas e respostas realizadas pelos estudantes, na sala de aula, e as respostas são enviadas para o professor por meio do App.

\subsection{Blogs e redes sociais}

Os blogs (ou weblog) e as redes sociais são sites que promovem a interatividade e o compartilhamento de conteúdo. As redes sociais ou redes de relacionamentos virtuais visam o impulsionamento das relações 
humanas através da tecnologia. Essas tecnologias permitem a construção de conhecimento por meio de plataformas virtuais, alargando o tempo em que a aprendizagem pode ocorrer. ${ }^{34}$ Essas duas ferramentas interligam-se através da divulgação e difusão de informações contidas nos blogs pelas redes sociais, por meio de pequenos posts.

Existem diversos blogs específicos em diferentes campos da ciência. Alguns blogs que abordam conteúdos relacionados à Química, são: Química Periódica, ${ }^{35}$ Ensino de Química, ${ }^{36}$ Quimilokos, ${ }^{37}$ Ensino Virtual de Química, ${ }^{38} \mathrm{~A}$ graça da Química, ${ }^{39}$ entre outros.

Raupp e Eichler, ${ }^{40}$ listam três páginas e oito comunidades do Facebook, as quais têm como proposito divulgar os seus blogs, sendo elas:

- TabelaPeriódica.Org

(https://www.facebook.com/tabelaperiodica. org/);

- Diário de um Químico Digital (https://www.facebook.com/digimarcio/);

- Ensino de Química (https://www.facebook.com/Ensino-deQu\%C3\%ADmica-168613099852007/);

- Química Ensinada (https://www.facebook.com/quimicaensinad a/);

- Química Anormal (https://www.facebook.com/qu1.mic4/);

- Química da Depressão (https://www.facebook.com/quimicadadepre ssaoreal/);

- Quimicando

(https://www.facebook.com/Qu\%C3\%ADmic ando-618361391597777/) e

- Canal Fala Química (https://www.facebook.com/falaQuimica/).

Os autores consideram o "Canal Fala Química" como sendo o maior canal de divulgação da ciência e pesquisa química no Facebook. Evidenciam que acreditam na aplicação dos blogs na área de ensino como meios de repositórios de informações, que podem promover debates e discussões, ajudar na construção do conhecimento, criar uma aprendizagem reflexiva, gerar habilidades de leitura e escrita, promover o letramento digital, entre outras. Ao final divulgam a comunidade que criaram no Facebook, "Cientista Didático", a qual difunde os seguintes assuntos: conceitos específicos, resenha, biografia ou perfil, metodologia e entrevista.

\section{Discussão}

Como resultado das pesquisas realizadas, percebe-se que existem sugestões relevantes quanto ao uso dessas ferramentas tecnológicas no ensino de Química, sendo que as TICs podem ser utilizadas como aliadas no ensino tradicional. Para isso, é necessário que o docente desta disciplina se atente às tecnologias disponíveis a fim de usufruir dos seus benefícios, como deixar a aula mais dinâmica e interessante.

Sabe-se que os alunos do século XXI são ditos como geração $Z$ (nativos digitais). Eles estão ligados intimamente à expansão exponencial da internet e dos aparelhos tecnológicos, estando muito familiarizados com a world wide web, com o compartilhamento de arquivos, com os smartphones e tablets, passando uma boa parte do tempo conectados a essas tecnologias. ${ }^{41} \mathrm{O}$ uso desta tecnologia em sala de aula precisa ser empregada sem perder o foco principal, que é o ensino diferenciado e ativo do aluno, porém, caso este foco seja perdido, o uso do smartphone pode ser visto como um inimigo, que distrai e tira a atenção do aluno daquilo que é apresentado em sala de aula. ${ }^{42}$

Nesse sentido, o docente pode utilizar desses recursos de forma consciente como um grande aliado em suas aulas, inserindo-os, inclusive, nos planejamentos para as aulas de química, pois inúmeras ferramentas são disponibilizadas de forma gratuita, 
necessitando apenas, do interesse e incentivo, por parte do docente e instituição em utilizálas.

Porém, é preciso levar em consideração as dificuldades que os professores, tanto do ensino médio como do superior, infelizmente, ainda possuem para utilizar de tais recursos para complementar os conteúdos de química em sala de aula. Gaião et al., ${ }^{43}$ afirmam que "uma das principais dificuldades é devido ao pouco incentivo à incorporação de tecnologias nos currículos dos cursos de formação inicial (licenciaturas)". Nesse contexto, é fundamental que as instituições de ensino, que ainda não oferecem, incluam na formação docente conteúdos que abordem as tecnologias, dando destaque àquelas de que os nativos digitais fazem mais uso, como uma forma de acompanhar e compreender esse público no qual o docente lidará em sua vida profissional. Além de um maior incentivo governamental relacionado a estas tecnologias.

No ensino médio, a dificuldade é ainda maior, muitas escolas ainda não disponibilizam de computadores e internet, nem mesmo de datashow. Sendo assim, a não utilização de ferramentas tecnológicas, muitas vezes, está mais relacionada a falta de infraestrutura do que a falta de iniciativa do professor.

De fato, é importante a formação continuada desses profissionais, procurando conhecer as tecnologias de informação, assim como compreender e aprender mais sobre as metodologias pedagógicas. Dificilmente, em disciplinas de graduação e pós-graduação, que não sejam específicas ao tema abordado, será exigido por parte do programa que o professor tenha o domínio dessas novas tecnologias, embora, a falta de aprimoramento da mesma irá deixá-lo distante da realidade de seus alunos. Isso se aplica ao ensino médio também, que por mais que a escola ofereça recursos tecnológicos, fica a critério do professor utilizá-lo ou não.

Como afirma Gresczysczyn, Camargo Filho e Monteiro: ${ }^{30}$
O trabalho do docente não consiste apenas na transmissão de informações, de conhecimentos $e$ de saberes, mas em apresentá-los de forma inovadora, estimulante e consistente, com o propósito de que a aprendizagem, através do uso da tecnologia, possibilite um fazer educacional, em que sejam trabalhados os conteúdos de forma interativa, colaborativa e prazerosa, tendo como eixo norteador o contexto sóciopolítico-cultural em que o aluno está inserido (p. 401).

O foco, portanto, não é que o professor domine todas ferramentas tecnológicas, mas que tenha um mínimo de conhecimento para que seja suficiente o uso estratégico desse recurso para suas aulas.

\section{Conclusão}

De acordo com o exposto neste trabalho, o docente de Química pode ter como aliado o uso de tecnologias que estão sempre à mão dos alunos e que se tornam essenciais em sua rotina de comunicação e interatividade. São disponibilizadas várias ferramentas gratuitas para aulas teóricas e práticas desse conteúdo, podendo servir como uma espécie de "laboratório virtual" para muitas escolas que não possuem laboratório físico para o ensino prático da química, quando se fala em nível médio. Posto isso, é preciso que o docente, tanto da educação básica como da superior, desperte para a utilização desses recursos tecnológicos, uma vez que se torna necessário sair do tradicionalismo, mostrando o interesse pela aprendizagem do aluno, bem como, preocupação pela sua própria capacitação para manuseio de tais métodos. Gradativamente, discutir, questionar e cobrar das instituições de formação docente, enquanto forem acadêmicos, sobre a relevância do uso e inserção dessas tecnologias em sala de aula, pois, como foi visto no levantamento bibliográfico, o uso dessas TICs, desperta o interesse do aluno e 
também melhora o processo de ensinoaprendizagem.

\section{Referências Bibliográficas}

${ }^{1}$ Meira, M. E. M. Desenvolvimento e aprendizagem: reflexões sobre suas relações e implicações para a prática docente. Ciência \& Educação 1998, 5, 61. [CrossRef]

${ }^{2}$ Freire, E. P. A. O trabalho de informação na sociedade do aprendizado contínuo. Informação \& Sociedade: Estudos 2007, 17, 39. [Link]

${ }^{3}$ Sancho, J. M.; Tecnologias para transformar a educação, 1a. ed., Artmed: Porto Alegre, 2006.

${ }^{4}$ Moran Costa, J. M.; Masetto, M. T.; Behrens, M. A.; Novas Tecnologias e Mediação Pedagógica, 21a. ed., Papirus: Campinas, 2013.

${ }^{5}$ Libâneo, J. C.; Suanno, M. V. R.; Didática e escola em uma sociedade complexa. 1a. ed., CEPED: Goiânia, 2011.

${ }^{6}$ Kenski, V. M. Educação e tecnologias: o novo ritmo da informação, 1a. ed., Campinas, SP: Ed. Papirus, 2012.

${ }^{7}$ Nilson, L. L.; Stochero, A. D.; Forrati, S. M. Reflexões sobre o uso das tecnologias de informação e comunicação como ferramenta auxiliar no processo de ensino e aprendizagem. Anais do EATI 2017, 70, 207. [Link]

${ }^{8}$ Almeida, M. E. B. Educação a distância na internet: abordagens e contribuições dos ambientes digitais de aprendizagem. Educação e Pesquisa 2003, 29, 327. [CrossRef]

${ }^{9}$ Lins, G. S. A tecnologia e cultura de informação como cenário de pesquisa para a ciência. Biblios 2015, 6, 85. [CrossRef]

${ }^{10}$ Ferreira, V. F. As tecnologias interativas no ensino. Química Nova 1998, 21, 780. [CrossRef]

${ }^{11}$ Batista, G. C.; Lima, A. R.; Crisóstomo, L. C. S.; Marinho, M. M.; Marinho, E. S. Softwares para o ensino de química: chemsketch ${ }^{\circledR}$ um poderoso recurso didático. Revista Educacional Interdisciplinar 2016, 5, 1. [Link]

12 Mendes, A. P.; Santana, G. P.; Pessoa Júnior, E. S. F. O uso de software PhET como ferramenta para o ensino de balanceamento de reação química. Revista Areté 2015, 8, 16, 52. [Link]

${ }^{13}$ Ribeiro, A. A.; Greca, I. M. Simulações computacionais e ferramentas de modelização em educação Química: uma revisão de literatura publicada. Química Nova 2003, 26, 542. [CrossRef]

${ }^{14}$ Locatelli, A.; ZOCH, A. N.; Trentin, M. A. S. TICs no Ensino de Química: Um Recorte do "Estado da Arte". Revista Tecnologias na Educação 2015, 7, 1. [Link]

${ }^{15}$ Soares-Leite, W. S.; Nascimento-Ribeio, C. A. A inclusão das TICs na educação brasileira: problemas e desafios. Magis, Revista Internacional de Investigación en Educación 2012, 5, 173. [Link]

${ }^{16}$ Colossi, N.; Consentino, A.; Queiroz, E. G. Mudanças no contexto do Ensino Superior no Brasil: Uma tendência ao ensino colaborativo. Revista da FAE 2001, 4, 49. [Link]

${ }^{17}$ Gil, A.C. Como elaborar projetos de pesquisa. São Paulo: Atlas, 2007.

${ }^{18}$ Silva, G. N.; Dantas Filho, F. F.; Andrade, R. B. Ensino de Química e Química Computacional: teoria do estado de transição. Revista Tecnologias na Educação 2016, 17, 1. [Link]

${ }^{19}$ Lima, E. F. O Uso das TICs e da Pesquisa como Recursos Pedagógicos no Ensino de Bioquímica para o Curso de Licenciatura em Química. Revista de Graduação USP 2017, 2, 115. [CrossRef]

${ }^{20}$ Pauletti, F.; Mendes, M.; Rosa, M. P. A.; Catelli, F. Ensino de química mediado por tecnologias digitais: o que pensam os professores brasileiros? Revista Interaç̧ões 2017, 13, 144. [CrossRef]

${ }^{21}$ Silva, G. R.; Gandra, L. P. Aplicação de um roteiro exploratório para o uso da simulação computacional: modelos do átomo de hidrogênio. Revista Electrónica Formación y Calidad Educativa 2017, 5, 17. [Link] 
22 Ramos, C. M.; Ramos, L. A.; Lobato, C. C.; Hage Melim, L. I. S.; Santos, C. B. R. Modelagem Molecular como Ferramenta Motivadora no Ensino-Aprendizagem em Mecanismos de Reações de DielsAlder. Revista Virtual Química 2016, 8, 2026. [CrossRef]

${ }^{23}$ Raupp, D.; Serrano, A.; Moreira, M. A. Desenvolvendo habilidades visuoespaciais: uso de software de construção de modelos moleculares no ensino de isomeria geométrica em Química. Experiências em Ensino de Ciências 2009, 4, 65. [Link]

${ }^{24}$ Freire, E. P. A. Podcast: breve história de uma nova tecnologia educacional. Educação em Revista 2017, 18, 55. [Link]

${ }^{25}$ Leite, B. S.; Leão, M. B. C.; Andrade, S. A. Videocast: uma abordagem sobre pilhas eletrolíticas no ensino de química. Revista Tecnologias na Educação 2010, 2, 1. [Link]

${ }^{26}$ Felder, R. M. Learning and teaching styles in engineering education Journal of Engineering Education 1988, 78, 674. [Link]

${ }^{27}$ Freire, E. P. A. O podcast como ferramenta de educação inclusiva para deficientes visuais e auditivos. Revista Educação Especial 2011, 4, 195. [Link]

${ }^{28}$ Leite. B. S. Podcasts in the Chemistry Teaching. Orbital: The Electronic Journal of Chemistry 2016, 8, 341. [CrossRef]

29 Trigo, M. V. A. Dissertação de Mestrado, Pontifícia Universidade Católica de São Paulo, 2014. [Link]

${ }^{30}$ Gresczysczyn, M. C. C; Camargo Filho, P. S.; Monteiro, E. L. Aplicativos Educacionais para Smartphone e sua Integração Com o Ensino de Química. Revista de Ensino, Educação. E Ciência 2016, 17, 398. [CrossRef]

${ }^{31}$ Silva, P. F.; Silva, T. P.; Silva, G. N. StudyLab: Construção e Avaliação de um aplicativo para auxiliar o Ensino de Química por professores da Educação Básica. Revista Tecnologias na Educação 2015, 7, 1. [Link]

32 Oliveira, F. C.; Souto, D. L. P.; Carvalho, J. W. $P$. Seleção e análise de aplicativos com potencial para o ensino de química orgânica.
Revista Tecnologias na Educação 2016, 17, 1.

[Link]

${ }^{33}$ Nichele, A. G.; Canto, L. Z. Ensino de Química com Smartphones e Tablets. Novas Tecnologias na Educação 2016, 14, 1. [CrossRef]

${ }^{34}$ Coutinho, C. P.; Bottentuit Júnior, J. B. Collaborative learning using wiki: a pilot study with master students in educational technology in Portugal. Proceedings of World Conference on Educational Multimedia, Hypermedia e Telecommunications 2007, 1786. [Link]

${ }^{35}$ Química Periódica. Disponível em: < https://www.marquecomx.com.br/>. Acesso em: 21 maio 2019.

${ }^{36}$ Ensino de Química. Disponível em: < https://ensquimica.blogspot.com/?fbclid=Iw AR2jfOX7uS7Kh6wH1PgAmTCfmfpof-

D44J5KZkGA6gfx5f3hVfwAAywrBLQ>. Acesso em: 21 maio 2019.

${ }^{37}$ Quimilokos. Disponível em: $<$ https://quimilokos.blogspot.com/?fbclid=Iw ARO-

Ve5kfmRcNVaHvvNvlpaD5RRmYeCRFgXOTZg W6ukHC1NJcyoEnM2I53Y>. Acesso em: 21 maio 2019.

${ }^{38}$ Ensino Virtual de Química. Disponível em: $<$ https://ensinovirtualdequimica.blogspot.co m/>. Acesso em: 21 maio 2019.

${ }^{39}$ A graça da Química. Disponível em: <http://agracadaquimica.com.br/>. Acesso em: 21 maio 2019.

${ }^{40}$ Raupp, D.; Eichler, M. L. A rede social Facebook e suas aplicações no ensino de química. Novas Tecnologias na Educação 2012, 10, 1. [Link]

${ }^{41}$ Silva Júnior, W. F. Dissertação de Mestrado, Centro Universitário Salesiano de São Paulo, 2017. [Link]

${ }^{42}$ Rodrigues, A. I.; Zatz, F. A escola contemporânea e o diálogo com a Geração Z: Estudo de caso de educomunicação no ensino médio do Colégio Israelita Brasileiro. Revista de Educação, Cultura e Comunicação 2016, 7, 37. [Link] 
${ }^{43}$ Gaião, O. T. V.; Jacon, L. S. C.; Oliveira, A. C. G.; Mello, I. C. A mediação de diálogos com heterogeneidade de linguagens entre Formadores de Professores e o processo de construção de um aplicativo móvel para o Ensino de Química. Revista Internacional de Aprendizaje em Ciencia, Matemáticas y Tecnología 2016, 3, 1. [Link] 